\title{
FORMULAÇÃO DE POMADA COM POTENCIAL ANTIOXIDANTE À BASE DO EXTRATO FOLIAR DE AROEIRA (Schinus terebinthifolius Raddi)
}

\author{
Maria Giuliane Gonçalves da Silva ${ }^{1 *}$, Rafael Jorge Santos Aracati Padilha ${ }^{1}$, Beatriz Santana Rocha ${ }^{1}$, Marília Grasielly \\ de Farias Silva ${ }^{1}$, Edson Renan Barros de Santana ${ }^{1}$, Kêsia Xisto da Fonseca Ribeiro de Sena ${ }^{2}$, Ricardo Yara ${ }^{3}$, Cláudia \\ Sampaio de Andrade Lima ${ }^{1}$
}

\author{
${ }^{1}$ Laboratório de Biofísica Química, UFPE; ${ }^{2}$ Laboratório de Antibióticos, UFPE; ${ }^{3}$ Laboratório de Engenharia Biomédica, UFPE \\ *maria.giuliane@hotmail.com
}

\section{INTRODUÇÃO}

A fitocosmética é o segmento da ciência cosmetológica que se dedica ao estudo e à aplicação dos conhecimentos da ação dos princípios ativos extraídos dos vegetais, em proveito da higiene, da estética, da correção e da manutenção de um estado normal e sadio da pele e do cabelo (ARAÚJO et al., 2010). Trata-se de uma área que, atualmente, tem-se dado grande enfoque, devida a busca por fontes alternativas renováveis, especialmente no Brasil, que tem a oportunidade de entrar em mercados bilioná rios em virtude da sua riqueza em recursos naturais.

A aroeira da praia, popularmente conhecida como Schinus terebinthifolius Raddi é uma espécie vegetal pertencente à família Anacardiaceae, nativa da América do Sul, especialmente Brasil, Argentina e Paraguai; em território brasileiro, é encontrada desde Pernambuco até o Rio Grande do Sul (Carvalho, 1994). A aroeira tem sido alvo de pesquisas envolvendo o uso de extratos de cascas, folhas e frutos, e do óleo essencial no auxílio a processos cicatriciais (Branco-Neto et al., 2006; Ribas et al., 2006; Estevão et al. 2013), como agente antibacteriano e antifúngico (Siddiqui et al., 1995; Siddiqui et al., 1996). De maneira similar, estudos relatam o potencial de uso da aroeira devido a sua atividade antiinflamatória (FORMAGIO et al., 2011), cicatrizante (NUNES JR et al., 2006), antitumoral (BENDAOUD et al., 2010), fungicida e no tratamento de úlceras (CARLINI et al., 2010), o que encoraja o aproveitamento dos extratos na formulação de pomadas dermatoló gicas comerciais.

Nesse sentido, o objetivo do presente estudo foi desenvolver uma pomada à base do extrato de Schinus terebinthifolius Raddi, assim como realizar uma análise antioxidante e antimicrobiana da planta em questão.

\section{MATERIAIS E MÉTODOS}

Schinus terebinthifolius Raddi foi coletada no campus Recife da Universidade Federal de Pernambuco. 0 material vegetal foi acondicionado em estufa, com circulação de ar forçada a $42^{\circ} \mathrm{C}$, para secagem. Posteriormente, as folhas da aroeira foram separadas para realizar o processo de trituração. 0 pó obtido foi colocado em um percolador de aço inoxidável, utilizando-se uma solução hidroalcoólica $(70 \%)$ no extrator. Para eliminação total do solvente, o extrato bruto foi concentrado em evaporador rotativo, a temperatura de $45^{\circ} \mathrm{C}$, e posteriormente levado ao dessecador até obter peso constante.

A confecção das pomadas foi realizada da seguinte forma: foram preparadas quatro pomadas, utilizando uma quantidade fixa de base $(1,25 \mathrm{~g}$ de lanolina anidra e $1,25 \mathrm{~g}$ de vaselina sólida) e variável de extrato. Para formulação da pomada $\mathrm{P} 1$, foram misturadas à base $2,5 \mathrm{~g}$ do extrato de aroeira, e 1,25, 0,25 e 0,125 para $\mathrm{P} 2, \mathrm{P} 3$ e $\mathrm{P} 4$, respectivamente. Um fitocosmético de aroeira comercializado por uma Comunidade Produtoras de Plantas Medicinais e fitoterápicas em Pernambuco foi utilizada para analise comparativa

A avaliação antioxidante foi realizada através do teste da redução do radical DPPH (2,2-difenil-1-picril-hidrazila), seguindo a metodologia proposta por Nascimento e colaboradores (2011), foi determinada a atividade antioxidante do extrato bruto. A mesma metodologia foi aplicada foi aplicada para as quatro formulações e para o fitocosmético. As atividades individuais de lanolina anidra e vaselina sólida também foram investigadas. As concentrações de extrato testadas foram de 1000, 500, 250, 125 e $62,5 \mu \mathrm{g} / \mathrm{mL}$. Todos os testes foram realizados em triplicata. Para as formulações, o fitocosmético, a lanolina e a vaselina foram preparadas concentraç ões de $1000 \mu \mathrm{g} / \mathrm{mL}$. Adicionou-se a cada concentração uma solução de DPPH $300 \mu \mathrm{M}$, exceto nos brancos, onde foi adicionado o solvente. 0 controle negativo foi feito pela adição de etanol e DPPH e o controle positivo foi feito pela adição de solução de um padrão (ácido ascórbico) e DPPH. Após a adição do reagente, esperou-se 30 minutos e procedeu-se a leitura no espectrofotô metro a $515 \mathrm{~nm}$. A porcentagem de atividade antioxidante foi obtida pela seguinte equação:

\section{Eliminação [DPPH] $(\%)=\underline{(\text { Abs amostra }- \text { Abs controle })} \times 100$}

Abs controle

Onde: Abs ( Absorbância)

Os testes para a determinação da atividade antimicrobiana foram realizados de acordo com o método de difusã o em disco (BAUER et al., 1966), com modificações, no laboratório do Departamento de Antibióticos da UFPE (UFPEDA), com os seguintes micro-organismos: Staphylococcus aureus (UFPEDA 01), Mycrococcus luteus (UFPEDA 06), Bacillus subtilis (UFPEDA 16), Pseudomonas aeruginosa (UFPEDA 39), Mycobacterium smegmatis (UFPEDA 71), Enterococcus faecalis (UFPEDA 138), Escherichia coli (UFPEDA 224), e a levedura Candida albicans (UFPEDA 1007).

Foram utilizados $200 \mathrm{mg}$ do extrato, para a diluição foi utilizada uma solução de $2 \mathrm{mg} / \mathrm{ml}$ de DMSO, e distribuídos em discos de papel de filtro esterilizados de $6 \mathrm{~mm}$ de diâmetro. Os discos foram postos sobre o meio de cultura correspondente para cada micro-organismo, a suspensão do inóculo dos micro- 
organismos em solução salina correspondeu a 0,5 na escala de McFarland, sendo os micro-organismos semeados em meio ágar Muller-Hinton (g/L): extrato de carne 30; caseína hidrolisada 17,5; amido 1,5; agar 17 e $\mathrm{pH}$ final 7,3 e $\mathrm{GL}(\mathrm{g} / \mathrm{L})$ : peptona 10; extrato de carne 3; $\mathrm{NaCl} 5$; extrato de levedura 10; glicose ou dextrose 10; agar 15 e pH final de 7,0com um swab estéril, após, as placas foram colocadas em estufa a $45^{\circ} \mathrm{C}$ num período de 24 horas, e apó $s$, foi realizada a observação e medição dos halos. Os controles positivos utilizados foram: Ciclofosfamida (CTX), Cefoxitina (CFO) e Clorafenicol (CLO). O controle negativo foram placas com apenas os microorganismos. Os testes foram realizados em triplicata

\section{RESULTADOS E DISCUSSÃO}

Os resultados do teste antioxidante para o extrato bruto de Schinus terebinthifolius Raddi indicaram alto percentual de atividade antioxidante para a concentração de $1000 \mu \mathrm{g} / \mathrm{ml}$ $(86,56 \%)$, por esse motivo, foi comparado ao padrão ácido ascó rbico. Os valores estão apresentados na Tabela 1.

Tabela 1. Resultado do teste da atividade antioxidante pela redução do radical DPPH para o extrato de Schinus terebinthifolius Raddi e para o controle positivo (ácido ascórbico).

\begin{tabular}{ccc}
\hline $\begin{array}{c}\text { Concentração }(\mu \\
\mathrm{g} / \mathrm{ml})\end{array}$ & $\begin{array}{c}\text { Atividade antioxidante } \\
\text { (\%) extrato }\end{array}$ & $\begin{array}{c}\text { Atividade antioxidante } \\
\text { (\%) ácido ascórbico }\end{array}$ \\
\hline 1000 & 86,56 & 95,64 \\
500 & 84,2 & 95,85 \\
250 & 53,3 & 95,93 \\
125 & 30,85 & 96,85 \\
62,5 & 16,43 & 95,87 \\
\hline
\end{tabular}

Num ensaio qualitativo Ceruks e colaboradores (2007) descrevem a avaliação do potencial anti-radicalar de substâncias fenólicas isoladas a partir das folhas de S. terebinthifolius. A relac ão dos compostos fenólicos com a atividade antioxidante já é amplamente encontrada na literatura (DEGÁSPARI \& WASZCZYNSKYJ, 2004; ACHKAR et al., 2013).

$\mathrm{Na}$ Tabela 2, observa-se que a atividade antioxidante manteve-se alta quando foram analisadas as amostras das pomadas desenvolvidas, ou seja, o ato de incorporar o extrato à pomadabase não provocou queda significativa da atividade. Também é possível notar que o fitocosmético testado para comparação não apresentou atividade antioxidante frente o ensaio realizado.

Tabela 2. Resultado do teste da atividade antioxidante pela redução do radical DPPH para as pomadas desenvolvidas à base de Schinus terebinthifolius Raddi e para o fitocosmético.

\begin{tabular}{cccccc}
\hline $\begin{array}{c}\text { Concentração } \\
(\mu \mathrm{g} / \mathrm{ml})\end{array}$ & \multicolumn{5}{c}{$\begin{array}{c}\text { Atividade antioxidante } \\
(\%)\end{array}$} \\
\hline 1000 & $\begin{array}{c}\text { Fitocosmé } \\
\text { tico } \\
0\end{array}$ & P1 & P2 & P3 & P4 \\
& 76,05 & 76,71 & 75,59 & 61,51 \\
\hline
\end{tabular}

Na Tabela 3, é possível verificar que foi o extrato bruto de Schinus terebinthifolius Raddi responsável por conferir tal atividade às formulações, visto que a lanolina e a vaselina não apresentaram capacidade antioxidante significante.

Tabela 3. Resultado do teste da atividade antioxidante pela redução do radical DPPH para os constituintes da pomada-base (lanolina anidra e vaselina sólida).

\begin{tabular}{ccc}
\hline Concentração $(\mu \mathrm{g} / \mathrm{ml})$ & \multicolumn{2}{c}{ Atividade antioxidante $(\%)$} \\
\hline 1000 & Lanolina anidra & Vaselina sólida
\end{tabular}

(-) valor igual ou próximo a zero.

0 extrato de aroeira apresentara atividade antimicrobiana para todos os micro-organismos segundo Tabela 4 .
Tabela 4: Resultados da avaliação antimicrobiana

\begin{tabular}{c|c}
\hline Micro-organismos testados & $\begin{array}{c}\text { Diâmetro dos Halos de inibição } \\
(\mathbf{m m})\end{array}$ \\
\hline Staphylococcus aureus & 16 \\
\hline Mycrococcus luteus & 19 \\
\hline Bacillus subtilis & 11 \\
\hline Pseudomonas aeruginosa & 0 \\
\hline Pseudomonas aeruginosa & 14 \\
\hline Enterococcus faecalis & 13 \\
\hline Escherichia coli & 0 \\
\hline Candida albicans & 13 \\
\hline
\end{tabular}

As atividades biológicas encontradas neste trabalho podem ser conferidas a ação de metabólitos secundários já relatados na literatura tais como: alcaloides, glicosídeos cardiotô nicos, flavonoides e taninos hidrolisáveis tanto em extratos como em óleos essenciais (SILVA et al., 2015; Santana et al., 2012). Estes compostos podem estar relacionados com a ação biológica encontrada na espécie em questão, apresentando assim potencial interesse biotecnológico seja para a área agronômica, biomédica ou farmacêutica.

\section{CONCLUSÕES}

Os valores obtidos nesses ensaios in vitro mostram que a incorporação de extrato bruto de aroeira em uma pomada pode ser efetiva no reparo de feridas, tratamento para rugas e prevenção contra o envelhecimento precoce, devida a comprovação de sua atividade antioxidante. No entanto, estudos adicionais in vivo são necessários para confirmação do potencial antioxidante e antibacteriana assim como sua possível utilização em um fitocosmé tico.

Apoio:

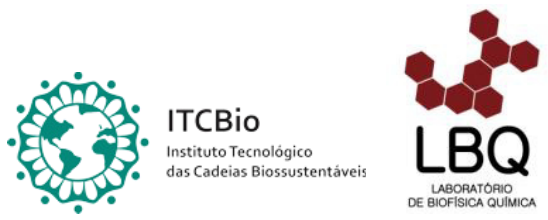

\section{REFERÊNCIAS}

ACHKAR, M. T.; NOVAES, G. M.; SILVA, M. J. D.; VILEGAS, W. Propriedade antioxidante de compostos fenólicos: importância na dieta e na conservação de alimentos. Revista da Universidade Vale do Rio Verde, v. 11, n. 2, p. 398-406, 2013.

ARAUJO, A. I. F. et. al. Plantas nativas do Brasil empregadas em fitocosmética. X Jornada de Ensino, Pesquisa e Extensão: 2010. Disponível

em: <http://www.sigeventos.com.br/jepex/inscricao/resumos/0001/R 0037-2.PDF>. Acesso em: 26/11/2017.

BAUER AW, Kirby WM, TURCK M. Antibiotic susceptibility testing by a standardized single disk method. Amer J Clin Pathol. 1966; 45 (32): 493- 6.

BENDAOUD, $\mathrm{H}$. et al. Chemical Composition and Anticancer and Antioxidant Activities of Schinus Molle L. and Schinus Terebinthifolius Raddi Berries Essential Oils. J. Food Sci., Chicago, v.75, n.6, p.C466-C472, 2010.

BRANCO NETO, M. L. C.; RIBAS FILHO, J. M.; MALAFAIA, O.; OLIVEIRA FILHO, M. A.; CZECZKO, N. G.; AOKI, S.; CUNHA, R.; FONSECA, M.; AGUIAR, L. R. F. Avaliação do extrato hidroalcoólico de aroeira (Schinus terebinthifolius Raddi) no processo de cicatrização de feridas de pele em ratos. Acta Cirúrgica Brasileira, v.21, p.17-21, 2006. Suplemento 6 . 
CARLINI, E. A. et al. Antiulcer effect of the pepper trees Schinus terebinthifolius Raddi (aroeira-da-praia) and Myracrodruon urundeuva Allemão, Anacardiaceae (aroeira-do-sertão). Braz. J. Pharm., Curitiba, v.20, n.2, p.140-146, 2010.

CARVALHO, P. E. R. Espécies florestais brasileiras: recomendações silviculturais, potencialidades e uso da madeira. Brasília: EMBRAPA, 1994.

CERUKS, M., ROMOFF, P., FÁVERO, O. A., LAGO, J. H. G. Constituintes fenólicos polares de Schinus terebinthifolius Raddi (Anacardiaceae). Química Nova, São Paulo, v.30, p.597-599, 2007.

DEGÁSPARI, C. H.; WASZCZYNSKYJ, N. Propriedades antioxidantes de compostos fenólicos. Visão Acadêmica, Curitiba, v. 5 (1), p. 3340, 2004.

ESTEVÃO, L. R. M.; MENDONÇA, F. S.; BARATELLA-EVÊNCIO, L.; SIMÕES, R. S., BARROS, M. E. G.; ARANTES, R. M. E.; RACHID, M. A.; EVÊNCIO-NETO, J. Effects of aroeira (Schinus terebinthifoliu Raddi) oil on cutaneous wound healing in rats. Acta Cirúrgica Brasileira, v. 28, n. 3, p. 202-209, 2013.

FORMAGIO, A. S. N. et al. Chemical Composition and AntiInflammatory Activity of the Essential Oil of Schinus terebinthifolius Raddi (Anacardiaceae) Fruits. Latin Am. J. Pharm., Buenos Aires v.30, n.8, p.1555- 1559, 2011.

NASCIMENTO, J. C.; LAGE, L. F. O.; CAMARGOS, C. R. D.; AMARAL, J. C.; COSTA, L. M.; SOUSA, N. A.; OLIVEIRA, F. Q. Determinação da atividade antioxidante pelo método DPPH e doseamento de flavonóides totais em extratos de folhas da Bauhinia variegata $L$. Rev. Bras. Farm., 2011; 92 (4): 327-332.

NUNES JR, J. A. T. et al. Evaluation of the hydro-alcoholic Schinus terebinthifolius raddi (Aroeira) extract in the healing process of the alba linea in rats. Acta Cir. Bras., São Paulo, v.21, n.SUPPL.3, p.8-15, 2006.

RIBAS, M. O.; SOUZA, M. H.; SARTORETTO, J.; T. A.; NORONHA, L.; ACRA, L. A. Efeito da Schinus terebinthifolius Raddi sobre o processo de reparo tecidual das lesões ulceradas induzidas na mucosa bucal do rato. Revista Odonto Ciência, v.21, n.53, p.245252, 2006.

SIDDIQUI, R.; ZAFAR, U.; CHAUDHRY, S. S.; AHMAD, H. Antimicrobial activity of essential oils from Schinus terebinthifolius, Cypress sempervirens, Citrus limon, Ferula assafoetida. Part I. Pakistan Journal of Scientific and Industrial Research, v. 38, n.9-10, p. 358-361, 1995.

SIDDIQUI, R.; AHMADA, H.; SULTANS, S.; EHTESHAMUDDIN, A.F.M.; SHIRREM, S. Antimicrobial activity of essential oils. Part II, Pakistan Journal of Scientific and Industrial Research, v.39, n.1-4, p.43-47, 1996.

SILVA, L. R.; OLIVERA, A. A.; LIMA, R. A. Identification of secondary metabolites ethanolic extract of leaves of Schinus terebinthifolius RADDI. South American Journal of Basci Education, Techinical and Technological, v. 2, n. 2, p. 84-93, 2015.

SANTANA, J. S.; SARTORELLI, P.; GUADAGNIN, R. C.; MATSUO, A. L.; FIGUEIREDO, C. R.; SOARES, M. G.; da SILVA, A. M.; LAGO, J. H. Essential oils from Shinus terebinthifolius leaves - chenical composition and vitro cytotoxicity evaluation. Pharm Biol., v. 50, n. 10, 2012. 